\title{
Improved nuclear matter calculations from chiral low-momentum interactions
}

\author{
K. Hebeler, ${ }^{1,2}$ S. K. Bogner, ${ }^{3}$ R. J. Furnstahl, ${ }^{2}$ A. Nogga,${ }^{4}$ and A. Schwenk ${ }^{5,6}$ \\ ${ }^{1}$ TRIUMF, 4004 Wesbrook Mall, Vancouver, BC, V6T 2A3, Canada \\ ${ }^{2}$ Department of Physics, The Ohio State University, Columbus, OH 43210, USA \\ ${ }^{3}$ National Superconducting Cyclotron Laboratory and Department of Physics and Astronomy, \\ Michigan State University, East Lansing, MI 48844, USA \\ ${ }^{4}$ Institute for Advanced Simulations, Institut für Kernphysik and Jülich Centre for Hadron Physics, \\ Forschungszentrum Jülich, 52425 Jülich, Germany \\ ${ }^{5}$ ExtreMe Matter Institute EMMI, GSI Helmholtzzentrum für Schwerionenforschung GmbH, 64291 Darmstadt, Germany \\ ${ }^{6}$ Institut für Kernphysik, Technische Universität Darmstadt, 64289 Darmstadt, Germany
}

\begin{abstract}
We present new nuclear matter calculations based on low-momentum interactions derived from chiral effective field theory potentials. The current calculations use an improved treatment of the three-nucleon force (3NF) contribution that includes a corrected combinatorial factor beyond Hartree-Fock that was omitted in previous nuclear matter calculations. We find realistic saturation properties using parameters fit only to few-body data, but with larger uncertainty estimates from cutoff dependence and the $3 \mathrm{NF}$ parametrization than in previous calculations.
\end{abstract}

PACS numbers: 21.65.-f, 21.30.-x, 21.60.Jz, 21.10.-Dr

Major advances in nuclear structure theory over the last decade have been made by expanding the reach of few-body calculations that use microscopic interactions between nucleons. This progress has unambiguously established the quantitative role of three-nucleon forces $(3 \mathrm{NF})$ for the ground state and spectra of light nuclei $(A \leqslant 12)[1,2]$. Pioneering extensions to larger nuclei reveal new facets of the $3 \mathrm{NF}$, such as its role in determining the location of the neutron dripline 3, 4] and in elucidating the doubly-magic nature of ${ }^{48} \mathrm{Ca}$ [5]. Pushing these successes to still heavier nuclei, which includes most of the table of nuclides, is a fundamental challenge for low-energy nuclear physics.

The historical route to heavy nuclei is through infinite nuclear matter, a theoretical uniform limit that first turns off the Coulomb interaction, which otherwise drives heavier stable nuclei toward an imbalance of neutrons over protons and eventually instability. However, predicting nuclear matter based on microscopic internucleon forces has proved to be an elusive target. In particular, few-body fits have not sufficiently constrained 3NF contributions around saturation density such that nuclear matter calculations are predictive. Nuclear matter saturation is very delicate, with the binding energy resulting from cancellations of much larger potential and kinetic energy contributions. When a quantitative reproduction of empirical saturation properties has been obtained, it was imposed by hand through adjusting short-range three-body forces (see, for example, Refs. [6, 7]).

The lack of progress toward controlled nuclear matter calculations has long been hindered by the difficulty of the nuclear many-body problem when conventional nuclear potentials are used. The present calculations continue an alternative approach to nuclear matter using soft Hamiltonians derived from interactions fit only to few-body $(A \leqslant 4)$ data. We find realistic satura- tion properties within our theoretical uncertainty bounds without adjustment of parameters. This progress follows by applying several recent developments: systematic starting Hamiltonians based on chiral effective field theory (EFT) [8], renormalization group (RG) methods [9] to soften the short-range repulsion and short-range tensor components of the initial chiral interactions so that convergence of many-body calculations is vastly accelerated 10-12], and a new 3NF fitting procedure to the ${ }^{4} \mathrm{He}$ radius rather than the binding energy [2]. (Alternative expansions using chiral interactions are described in Refs. 8, 13, 14]). The calculations here also employ an improved treatment of the $3 \mathrm{NF}$ contribution in manybody perturbation theory compared to Refs. [10, 15], which includes the full treatment of $3 \mathrm{NF}$ double-exchange diagrams and corrected 3NF combinatorial factors beyond Hartree-Fock. Note that previous calculations of neutron matter [16, 17] and finite nuclei [3, 5] are not affected.

Our results are summarized in Fig. 1, which shows the energy per particle of symmetric matter as a function of Fermi momentum $k_{\mathrm{F}}$, or the density $\rho=2 k_{\mathrm{F}}^{3} /\left(3 \pi^{2}\right)$. A grey square representing the empirical saturation point is shown in each of the nuclear matter figures. Its boundaries reflect the ranges of nuclear matter saturation properties predicted by phenomenological Skyrme energy functionals that most accurately reproduce properties of finite nuclei [18]. Although this determination cannot be completely model independent, the value is generally accepted for benchmarking infinite matter. In the future, calculations of the properties of finite nuclei will allow one to compare directly to experimental data.

The calculations of Fig. [1]start from the $\mathrm{N}^{3} \mathrm{LO}$ nucleonnucleon (NN) potential (EM $500 \mathrm{MeV}$ ) of Ref. [19]. This NN potential is RG-evolved to low-momentum interactions $V_{\text {low } k}$ with a smooth $n_{\exp }=4$ regulator [20]. For 


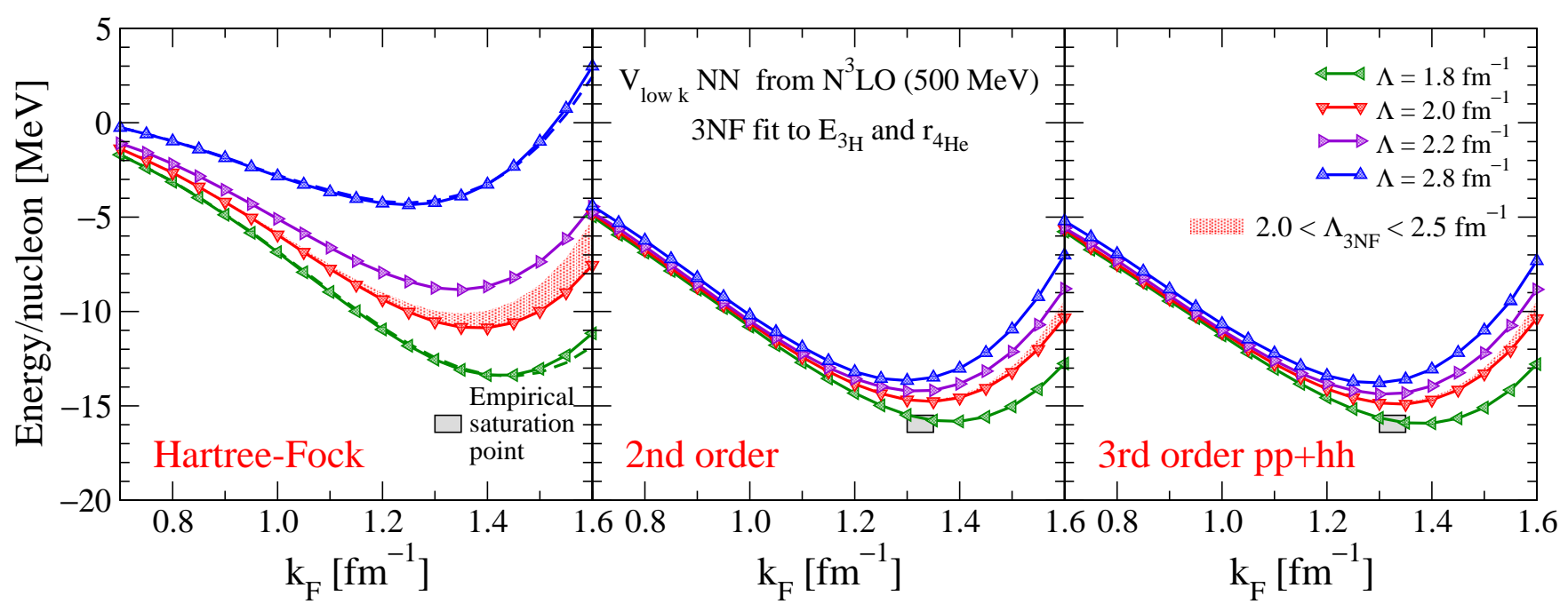

FIG. 1: (Color online) Nuclear matter energy per particle versus Fermi momentum $k_{\mathrm{F}}$ at the Hartree-Fock level (left) and including second-order (middle) and third-order particle-particle/hole-hole contributions (right), based on evolved $\mathrm{N}^{3} \mathrm{LO} \mathrm{NN}$ potentials and $3 \mathrm{NF}$ fit to $E_{3 \mathrm{H}}$ and $r_{{ }^{4} \mathrm{He}}$. Theoretical uncertainties are estimated by the NN (lines)/3N (band) cutoff variations.

each cutoff $\Lambda$, two couplings that determine the shorterrange parts of the $\mathrm{N}^{2} \mathrm{LO} 3 \mathrm{NF}$ 21] are fit to the ${ }^{3} \mathrm{H}$ binding energy and the ${ }^{4} \mathrm{He}$ matter radius using exact Faddeev and Faddeev-Yakubovsky methods as in Ref. [22]. Our $3 \mathrm{NF}$ central fit values are given in Table प we estimate that $c_{D}$ has an uncertainty of approximately 0.4 due to the uncertainties of the charge radius in ${ }^{4} \mathrm{He}$. We use a $3 \mathrm{NF}$ regulator of the form $\exp \left[-\left(\left(p^{2}+3 / 4 q^{2}\right) / \Lambda_{3 \mathrm{NF}}^{2}\right)^{n_{\exp }}\right]$ with $n_{\exp }=4$, where the $3 \mathrm{~N}$ cutoff $\Lambda_{3 \mathrm{NF}}$ is allowed to vary independently of the NN cutoff, which probes the sensitivity to short-range three-body physics. The shaded regions in Fig. 1 show the range of results for $2.0 \mathrm{fm}^{-1}<\Lambda_{3 \mathrm{NF}}<2.5 \mathrm{fm}^{-1}$ at fixed $\Lambda=2.0 \mathrm{fm}^{-1}$.

Nuclear matter is calculated in three approximations: Hartree-Fock (left), Hartree-Fock plus secondorder contributions (middle), and additionally summing third-order particle-particle and hole-hole contributions (right). The technical details regarding the treatment of the $3 \mathrm{NF}$ and the many-body calculation are as for neutron matter in Ref. [16]. We first construct a densitydependent two-body interaction from the $3 \mathrm{NF}$ by summing one particle over occupied states in the Fermi sea (see also Ref. 23]). This conversion simplifies the manybody calculation significantly and allows the inclusion of all 3NF double-exchange terms beyond Hartree-Fock, which were only approximated in Refs. 10, 15]. Furthermore, we have corrected the combinatorial factors at the normal-ordered two-body level of the 3NF from $1 / 6$ to $1 / 2$ in diagrams beyond Hartree-Fock used in these references (see Refs. [9, 16] for detailed discussions of these factors, which are correctly included in Refs. [3, [5, 16, 17]). To our knowledge, previous calculations in the literature of nuclear matter using normalordered 3NF contributions need the same correction.

The dashed lines in the left panel of Fig. 1) (for $\Lambda=$
1.8 MeV and $2.8 \mathrm{MeV}$ ) show the exact Hartree-Fock energy in comparison with the results obtained using the effective two-body interaction (solid lines). The excellent agreement supports the use of this density-dependent two-body approximation for symmetric nuclear matter. For the results beyond the Hartree-Fock level we use full momentum-dependent single-particle Hartree-Fock propagators. We have checked that the energies obtained using a self-consistent second-order spectrum overlap with the band of curves in Fig. 11

The Hartree-Fock results show that nuclear matter is bound even at this simplest level. A calculation without approximations should be independent of the cutoffs, so the spread in Fig. 1 1 sets the scale for omitted many-body contributions. The second-order results show a significant narrowing of this spread over a large density region. It is encouraging that our results agree with the empirical saturation point within the uncertainty in the many-body calculation and omitted higher-order manybody forces implied by the cutoff variation (the greater spread compared to Ref. [15] is mostly attributable to the corrected combinatorial factor). We stress that the cutoff dependence of order $3 \mathrm{MeV}$ around saturation density is small compared to the total size of the kinetic energy $(\approx 23 \mathrm{MeV})$ and potential energy $(\approx-38 \mathrm{MeV})$ at this density. Moreover, the cutoff dependence is smaller at $k_{\mathrm{F}} \approx 1.1 \mathrm{fm}^{-1}$, which resembles more the typical densities in medium-mass to heavy nuclei $\left(\rho=0.11 \mathrm{fm}^{-3}\right)$. For all cases in the right panel of Fig. 1, the compressibility $K=175-210 \mathrm{MeV}$ is in the empirical range.

The inclusion of third-order contributions gives only small changes from second order except at the lowest densities shown. This is consistent with nuclear matter being perturbative for low-momentum interactions, at least in the particle-particle channel [10]. The differ- 
ence at small densities is not surprising: the presence of a two-body bound state necessitates a nonperturbative summation in the dilute limit. We note that below saturation density, the ground state is not a uniform system, but breaks into clusters (see, for example, Ref. [24]).

In chiral EFT without explicit Deltas, $3 \mathrm{~N}$ interactions start at $\mathrm{N}^{2} \mathrm{LO}$ [21] and their contributions are given diagrammatically by

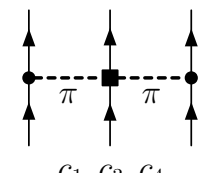

$$
c_{1}, c_{3}, c_{4}
$$

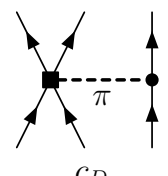

$c_{D}$

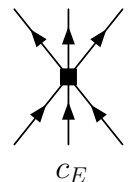

$c_{E}$
We assume that the $c_{i}$ coefficients of the long-range two-pion-exchange part are not modified by the RG. At present, we rely on the $\mathrm{N}^{2} \mathrm{LO} 3 \mathrm{NF}$ as a truncated "basis" for low-momentum $3 \mathrm{~N}$ interactions and determine the $c_{D}$ and $c_{E}$ couplings by a fit to data for all cutoffs [22]. In the future, fully evolved three- and four-body forces in momentum space starting from chiral EFT will be available (see Ref. 25] for an application of evolved $3 \mathrm{NF}$ in a harmonic-oscillator basis). The fit values of Table I are natural and the predicted ${ }^{4} \mathrm{He}$ binding energies are very reasonable. We have also verified that the resulting $3 \mathrm{NF}$ becomes perturbative in $A=3,4$ (see also Refs. [10, 15, 22]), i.e., the calculated individual 3NF contributions are largely unchanged if evaluated for wavefunctions using $\mathrm{NN}$ forces only.

The evolution of the cutoff $\Lambda$ to smaller values is accompanied by a shift of physics. In particular, effects due to iterated tensor interactions are replaced by threebody contributions. The role of the $3 \mathrm{NF}$ for saturation is demonstrated in Fig. 2. The two pairs of curves show the difference between the nuclear matter results for NN-only and $\mathrm{NN}$ plus $3 \mathrm{~N}$ interactions. It is evident that saturation is driven by the $3 \mathrm{NF}[10,15]$. Even for $\Lambda=2.8 \mathrm{fm}^{-1}$, which is similar to the lower cutoffs in chiral EFT potentials, saturation is at too high density without the $3 \mathrm{NF}$.

\begin{tabular}{|c|c|c|c|c|}
\hline & \multicolumn{2}{|c|}{$V_{\text {low } k}$} & \multicolumn{2}{|c|}{ SRG } \\
\hline$\Lambda$ or $\lambda / \Lambda_{3 \mathrm{NF}}\left[\mathrm{fm}^{-1}\right]$ & $c_{D}$ & $c_{E}$ & $c_{D}$ & $c_{E}$ \\
\hline $1.8 / 2.0\left(\mathrm{EM} c_{i}\right.$ 's $)$ & +1.621 & -0.143 & +1.264 & -0.120 \\
\hline $2.0 / 2.0\left(\mathrm{EM} c_{i}\right.$ 's $)$ & +1.705 & -0.109 & +1.271 & -0.131 \\
\hline $2.0 / 2.5\left(\mathrm{EM} c_{i}\right.$ 's $)$ & +0.230 & -0.538 & -0.292 & -0.592 \\
\hline $2.2 / 2.0\left(\mathrm{EM} c_{i}\right.$ 's $)$ & +1.575 & -0.102 & +1.214 & -0.137 \\
\hline $2.8 / 2.0\left(\mathrm{EM} c_{i}\right.$ 's $)$ & +1.463 & -0.029 & +1.278 & -0.078 \\
\hline $2.0 / 2.0\left(\mathrm{EGM} c_{i}{ }^{\prime} \mathrm{s}\right)$ & -4.381 & -1.126 & -4.828 & -1.152 \\
\hline $2.0 / 2.0\left(\mathrm{PWA} c_{i}\right.$ 's) & -2.632 & -0.677 & -3.007 & -0.686 \\
\hline
\end{tabular}

TABLE I: Results for the $c_{D}$ and $c_{E}$ couplings fit to $E_{3} \mathrm{H}=$ $-8.482 \mathrm{MeV}$ and to the point charge radius $r_{4} \mathrm{He}=1.464 \mathrm{fm}$ (based on Ref. 26]) for the NN/3N cutoffs and different $\mathrm{EM} / \mathrm{EGM} / \mathrm{PWA} c_{i}$ values used. For $V_{\text {low } k}$ (SRG) interactions, the $3 \mathrm{NF}$ fits lead to $E_{4 \mathrm{He}}=-28.22 \ldots-28.45 \mathrm{MeV}$ $(-28.53 \ldots-28.71 \mathrm{MeV})$.

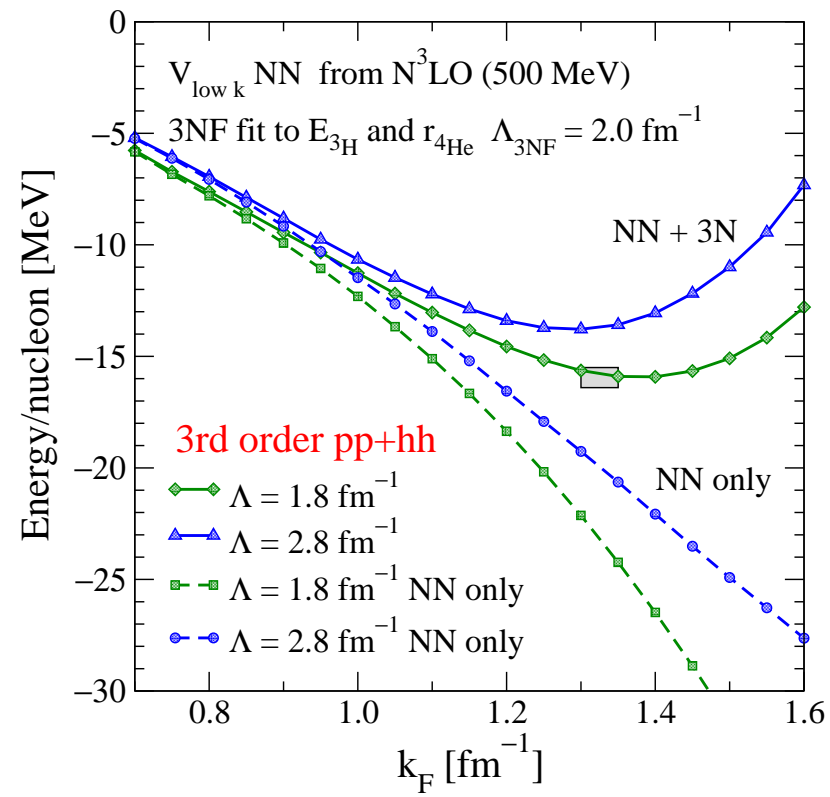

FIG. 2: (Color online) Nuclear matter energy of Fig. 1 at the third-order level compared to NN-only results for two representative NN cutoffs and a fixed $3 \mathrm{~N}$ cutoff.

Nevertheless, as in previous results [10, 15], the $3 \mathrm{~N}$ contributions and the $c_{D}, c_{E}$ fits are natural, and the same is expected for the ratio of three- to four-body contributions.

The smooth RG evolution used in the results so far is not the only choice for low-momentum interactions. A recent development is the use of flow equations to evolve Hamiltonians, which we refer to as the Similarity Renormalization Group (SRG) 27 29]. The flow parameter $\lambda$, which has dimensions of a momentum, is a measure of the degree of decoupling in momentum space. Fewbody results for roughly the same value of SRG $\lambda$ and smooth $V_{\text {low } k} \Lambda$ have been remarkably similar (see, for example, Ref. [1] ). With either RG method, we can also change the initial interaction. The results presented so far all start from a chiral EFT potential at a single order with one choice of EFT regulator implementation and values. There are several alternatives available [8, 19, 30], which are almost phase-shift equivalent (but the $\chi^{2}$ is not equally good up to $E_{\text {lab }} \approx 300 \mathrm{MeV}$ ). Universality for phase-shift equivalent chiral EFT potentials as $\Lambda$ decreases was shown for smooth-cutoff $V_{\text {low } k}$ interactions in Ref. [9, 20] in the form of the collapse of different initial potentials to the same matrix elements in each partial wave channel. An analogous collapse has been found for $\mathrm{N}^{3} \mathrm{LO}$ potentials evolved by the SRG to smaller $\lambda[9]$.

Based on this universal collapse for low-momentum interaction matrix elements it is natural to expect a similar collapse for the energy per particle in nuclear matter. We consider four different chiral NN potentials: the $\mathrm{N}^{3} \mathrm{LO}$ potential by Entem and Machleidt [19] for two different cutoffs 500 and $600 \mathrm{MeV}$, and the $\mathrm{N}^{3} \mathrm{LO} \mathrm{NN}$ potential 


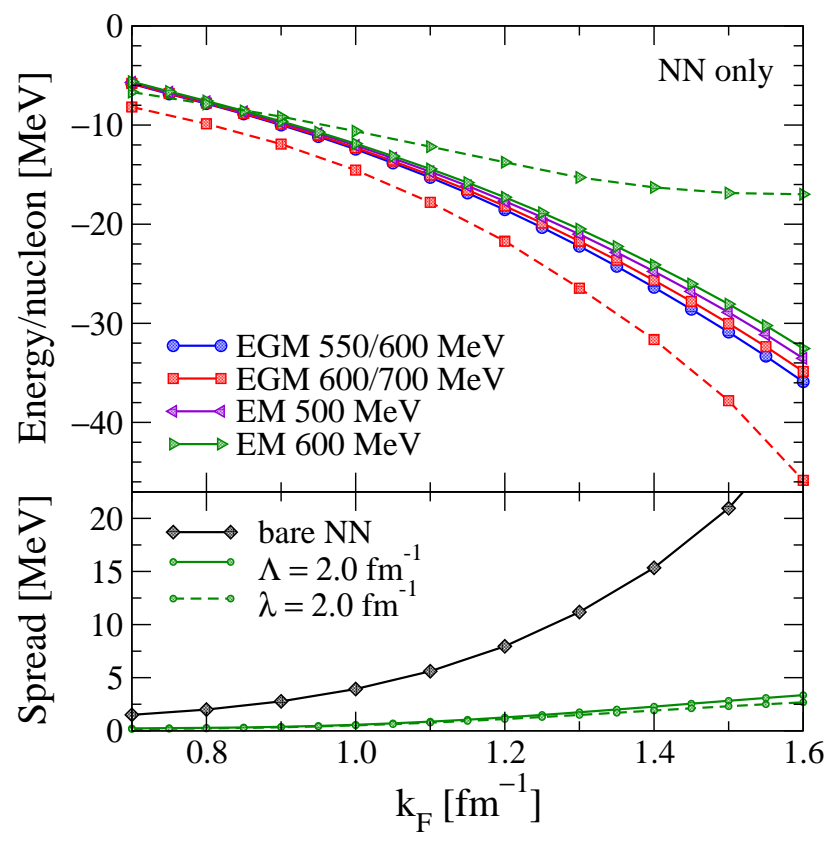

FIG. 3: (Color online) Nuclear matter NN-only results for different chiral $\mathrm{N}^{3} \mathrm{LO}$ potentials (EM [19] and EGM [30]). The upper panel shows the third-order results for $V_{\text {low } k \text {-evolved }}$ interactions at $\Lambda=2.0 \mathrm{fm}^{-1}$ (solid lines) and BruecknerHartree-Fock results for the two unevolved chiral potentials that provide the lowest and highest energies (dashed lines), EGM 600/700 MeV and EM $600 \mathrm{MeV}$. The lower panel shows the maximal spread of the energy results at these two cutoff scales $\Lambda$ for $V_{\text {low } k}$ and $\lambda$ for SRG-evolved NN interactions.

by Epelbaum et al. [30] (EGM) for two different cutoff combinations 550/600 MeV and 600/700 MeV. The results for the energy are presented in Fig. 3. The upper panel shows the energies for $V_{\text {low }} k \mathrm{NN}$-only interactions derived from different chiral NN potentials (solid lines) in comparison to Brueckner-Hartree-Fock (BHF, which means resummed particle-particle ladder) results based on unevolved chiral potentials (dashed lines). For clarity, we only display the two extreme BHF results. As shown in the lower panel we find a model dependence of about $13 \mathrm{MeV}$ for the unevolved $\mathrm{N}^{3} \mathrm{LO}$ potentials around saturation density and approximately $2 \mathrm{MeV}$ for the $V_{\text {low }} k$ and SRG low-momentum interactions, comparable to the cutoff variation in Fig. 1. The latter spread reflects the residual RG/SRG dependence on the initial interactions.

By supplementing the low-momentum NN interactions with corresponding 3NF's we can probe the sensitivity of the energy to uncertainties in the $c_{i}$ coefficients (see also Refs. 16, 31, 32]). We consider three different cases: first, we take low-momentum interactions evolved from the $\mathrm{N}^{3} \mathrm{LO} \mathrm{NN}$ potential EM $500 \mathrm{MeV}$ $\left(\mathrm{EM} c_{i}\right.$ 's: $c_{1}=-0.81 \mathrm{GeV}^{-1}, c_{3}=-3.2 \mathrm{GeV}^{-1}, c_{4}=$ $5.4 \mathrm{GeV}^{-1}$ ), second, low-momentum interactions from the EGM $550 / 600 \mathrm{MeV}$ potential (EGM $c_{i}$ 's: $c_{1}=$ $-0.81 \mathrm{GeV}^{-1}, c_{3}=-3.4 \mathrm{GeV}^{-1}, c_{4}=3.4 \mathrm{GeV}^{-1}$ ), and third, low-momentum interactions from the EM $500 \mathrm{MeV}$

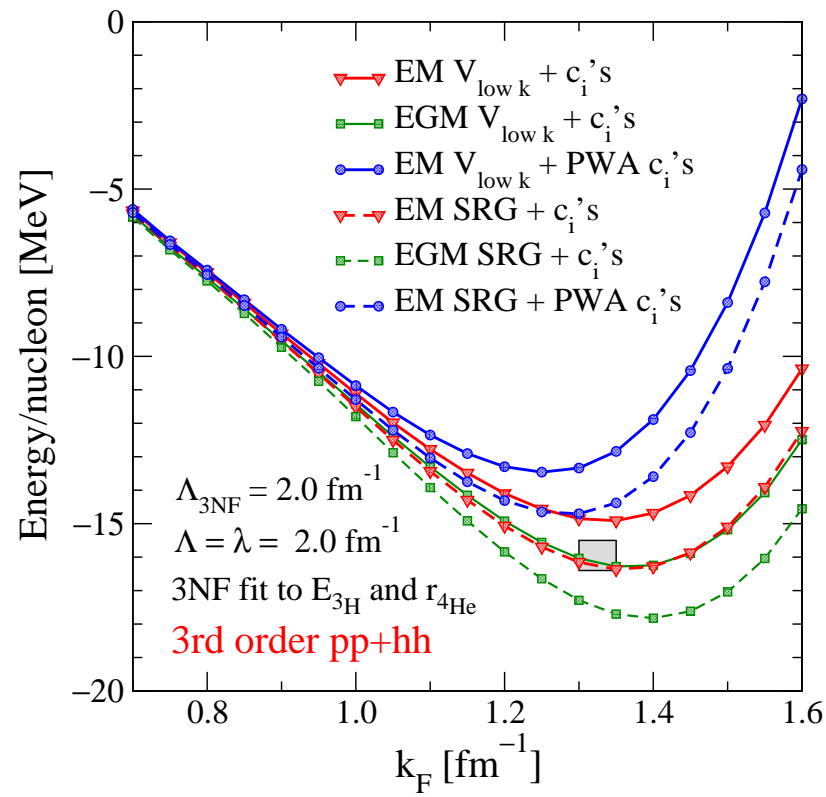

FIG. 4: (Color online) Nuclear matter energy at the thirdorder level comparing low-momentum $V_{\text {low } k}$ with SRGevolved chiral NN interactions for $3 \mathrm{NF}$ with different EM/PWA/EGM $c_{i}$ values used (see text).

potential combined with the central $c_{i}$ values obtained from the NN partial wave analysis [33] (PWA $c_{i}$ 's: $c_{1}=$ $\left.-0.76 \mathrm{GeV}^{-1}, c_{3}=-4.78 \mathrm{GeV}^{-1}, c_{4}=3.96 \mathrm{GeV}^{-1}\right)$. The fit values for $c_{D}$ and $c_{E}$ are given in Table 【.

The resulting nuclear matter energies are shown in Fig. 4. For all three cases we find realistic saturation properties within the theoretical uncertainties implied by the cutoff dependence shown in Fig. 1 and the NN interaction-dependence shown in Fig. 3. The difference between $V_{\text {low } k}$ and SRG results for a given set of $c_{i}$ is similar to the NN-only case (see Fig. 11), which helps support the general nature of the $3 \mathrm{NF}$ fit. However, the present sensitivity study can clearly only provide a first estimate for the energy spread due to uncertainties of the $c_{i}$ couplings. A more systematic study will require a correlation analysis based on a larger set of results.

The theoretical errors of our nuclear matter results arise from truncations in the initial chiral EFT Hamiltonian, the approximation of the $3 \mathrm{NF}$, and the manybody approximations. Corrections to the present calculation include higher-order many-body terms, in particular particle-hole corrections, and contributions from higherorder many-body forces and from $3 \mathrm{NF}$ contributions that cannot be expressed in terms of density-dependent twobody interactions. While the improvements in the cutoff dependence suggest that these corrections are relatively small, an approach such as coupled cluster theory that can perform a high-level resummation is ultimately necessary for a robust validation.

While nuclear matter has lost to light nuclei its status as the first step to nuclear structure, it is still key as a 
step to heavier nuclei and astrophysical applications like the structure of neutron stars [17]. Our results can help with efforts to develop ab-initio density functional theory (DFT) based on expanding about nuclear matter 34]. This is analogous to the application of DFT in quantum chemistry and condensed matter starting with the uniform electron gas in local-density approximations and adding constrained derivative corrections. Phenomenological energy functionals (such as Skyrme) for nuclei have impressive successes but lack a (quantitative) microscopic foundation based on nuclear forces and seem to have reached the limits of improvement with the current form of functionals [35, 36]. At present, the theoretical errors of our results, while small on the scale of the potential energy per particle, are too large to be quantitatively competitive with existing functionals. The implementation of higher-order chiral Hamiltonians and their RG evolution can be expected to provide more accurate and reliable predictions. However, there is also the possibility of fine tuning to heavy nuclei, of using EFT/RG to guide next-generation functional forms [37, 38], and of benchmarking with ab-initio methods for low-momentum interactions. Work in these directions is in progress.

In summary, we have presented new results for nuclear matter based on chiral NN and $3 \mathrm{~N}$ interactions with RG evolution. The chiral EFT framework provides a systematic improvable Hamiltonian while the softening of nuclear forces by RG evolution enhances the convergence and control of the many-body calculation. The empirical saturation point is reproduced within our estimates of uncertainties despite input only from few-body data. Because of the fine cancellations, however, significant reduction of these uncertainties will be needed before direct DFT calculations of nuclei are competitive. Nevertheless, these results are very promising for a unified description of all nuclei and nuclear matter.

We thank J. W. Holt for helpful discussions. This work was supported in part by NSERC, the NSF under Grant Nos. PHY-0653312, PHY-0758125 and PHY-1002478, the UNEDF SciDAC Collaboration under DOE Grant DE-FC02-07ER41457, the Helmholtz Alliance Program of the Helmholtz Association, contract HA216/EMMI "Extremes of Density and Temperature: Cosmic Matter in the Laboratory" and the DFG through grant SFB 634. TRIUMF receives funding via a contribution through the NRC Canada. Part of the numerical calculations have been performed at the JSC, Jülich, Germany.

[1] S. C. Pieper, R. B. Wiringa and J. Carlson, Phys. Rev. C 70, 054325 (2004); S. C. Pieper, Riv. Nuovo Cim. 031, 709 (2008).

[2] P. Navrátil, V. G. Gueorguiev, J. P. Vary, W. E. Ormand and A. Nogga, Phys. Rev. Lett. 99, 042501 (2007); P. Navrátil, S. Quaglioni, I. Stetcu and B. R. Barrett, J.
Phys. G 36083101 (2009).

[3] T. Otsuka, T. Suzuki, J. D. Holt, A. Schwenk and Y. Akaishi, Phys. Rev. Lett. 105, 032501 (2010).

[4] G. Hagen, T. Papenbrock, D. J. Dean, M. Hjorth-Jensen and B. Velamur Asokan, Phys. Rev. C 80, 021306 (2009).

[5] J. D. Holt, T. Otsuka, A. Schwenk and T. Suzuki, arXiv:1009.5984

[6] A. Akmal, V. R. Pandharipande and D. G. Ravenhall, Phys. Rev. C 58, 1804 (1998).

[7] A. Lejeune, U. Lombardo and W. Zuo, Phys. Lett. B 477, 45 (2000).

[8] E. Epelbaum, H.-W. Hammer and U.-G. Meißner, Rev. Mod. Phys. 81, 1773 (2009).

[9] S. K. Bogner, R. J. Furnstahl and A. Schwenk, Prog. Part. Nucl. Phys. 65, 94 (2010).

[10] S. K. Bogner, A. Schwenk, R. J. Furnstahl and A. Nogga, Nucl. Phys. A 763, 59 (2005).

[11] S. K. Bogner, R. J. Furnstahl, P. Maris, R. J. Perry, A. Schwenk and J. P. Vary, Nucl. Phys. A 801, 21 (2008).

[12] S. Bacca, A. Schwenk, G. Hagen and T. Papenbrock, Eur. Phys. J. A 42, 553 (2009).

[13] N. Kaiser, S. Fritsch and W. Weise, Nucl. Phys. A 697, 255 (2002).

[14] A. Lacour, J. A. Oller and U.-G. Meißner, Ann. Phys. 326, 241 (2011).

[15] S. K. Bogner, R. J. Furnstahl, A. Nogga and A. Schwenk, arXiv:0903.3366

[16] K. Hebeler and A. Schwenk, Phys. Rev. C 82, 014314 (2010).

[17] K. Hebeler, J. M. Lattimer, C. J. Pethick and A. Schwenk, Phys. Rev. Lett. 105, 161102 (2010).

[18] M. Bender, P.-H. Heenen and P.-G. Reinhard, Rev. Mod. Phys. 75, 121 (2003).

[19] D. R. Entem and R. Machleidt, Phys. Rev. C 68, 041001(R) (2003).

[20] S. K. Bogner, R. J. Furnstahl, S. Ramanan and A. Schwenk, Nucl. Phys. A 784, 79 (2007); K. Hebeler, A. Schwenk and B. Friman, Phys. Lett. B 648, 176 (2007).

[21] U. van Kolck, Phys. Rev. C 49, 2932 (1994); E. Epelbaum, A. Nogga, W. Glöckle, H. Kamada, U.-G. Meißner and H. Witala, Phys. Rev. C 66, 064001 (2002).

[22] A. Nogga, S. K. Bogner and A. Schwenk, Phys. Rev. C 70, 061002(R) (2004).

[23] J. W. Holt, N. Kaiser and W. Weise, Phys. Rev. C 81, $024002(2010)$.

[24] C. J. Horowitz and A. Schwenk, Nucl. Phys. A 776, 55 (2006).

[25] S. K. Bogner, R. J. Furnstahl and R. J. Perry, Ann. Phys. 323, 1478 (2008); E. D. Jurgenson, P. Navratil and R. J. Furnstahl, Phys. Rev. Lett. 103, 082501 (2009).

[26] I. Sick, Phys. Rev. C 77, 041302 (2008).

[27] S. D. Glazek and K. G. Wilson, Phys. Rev. D 48, 5863 (1993); F. Wegner, Ann. Phys. (Leipzig) 3, 77 (1994).

[28] S. K. Bogner, R. J. Furnstahl and R. J. Perry, Phys. Rev. C 75, 061001(R) (2007).

[29] An alternative non-RG use of unitary transformations to reduce correlations in many-body wave functions is described in R. Roth, H. Hergert, P. Papakonstantinou, T. Neff and H. Feldmeier, Phys. Rev. C 72, 034002 (2005), and references therein.

[30] E. Epelbaum, W. Glöckle and U.-G. Meißner, Nucl. Phys. A 747, 362 (2005).

[31] L. Tolos, B. Friman and A. Schwenk, Nucl. Phys. A 806, 105 (2008). 
[32] V. Bernard, Prog. Part. Nucl. Phys. 60, 82 (2008).

[33] M. C. M. Rentmeester, R. G. E. Timmermans and J. J. de Swart, Phys. Rev. C 67, 044001 (2003).

[34] S. K. Bogner, R. J. Furnstahl and L. Platter, Eur. Phys. J. A 39, 219 (2009). B. Gebremariam, T. Duguet and S. K. Bogner, Phys. Rev. C 82, 014305 (2010).

[35] G. F. Bertsch, B. Sabbey and M. Uusnakki, Phys. Rev. C 71, 054311 (2005).
[36] M. Kortelainen, J. Dobaczewski, K. Mizuyama and J. Toivanen, Phys. Rev. C 77, 064307 (2008).

[37] B. Gebremariam, S. K. Bogner and T. Duguet, Nucl. Phys. A 851, 17 (2011)

[38] M. Stoitsov, M. Kortelainen, S. K. Bogner, T. Duguet, R. J. Furnstahl, B. Gebremariam and N. Schunck, Phys. Rev. C 82, 054307 (2010). 